\section{Kikuchi-Fujimoto disease involving retroperitoneal lymph nodes: An uncommon presentation}

\author{
Jane Date Hon, ${ }^{1}$ Maria E. Vergara- \\ Lluri, ${ }^{1}$ Imran Siddiqi, ${ }^{1}$ Christopher \\ Foss, ${ }^{2}$ Donald I. Feinstein, ${ }^{2}$ \\ Russell K. Brynes ${ }^{1}$ \\ ${ }^{1}$ Department of Pathology and \\ Hematology Division, ${ }^{2}$ Department of \\ Medicine, University of Southern \\ California Keck School of Medicine, Los \\ Angeles, CA, USA
}

\begin{abstract}
Kikuchi-Fujimoto disease is a self-limited disease of unknown etiology that is clinically defined by fevers accompanied by tender posterior cervical lymphadenopathy. It often presents acutely or sub-acutely, and due to its non-specific features, the differential diagnosis is broad and includes infectious, autoimmune, and malignant causes. Although cases of extra-cervical disease are not uncommon, involvement of retroperitoneal lymph nodes has only rarely been reported. Here, we describe a patient with Kikuchi-Fujimoto disease who presented with fever of unknown origin, abdominal pain, and enlarged hypermetabolic retroperitoneal lymph nodes.
\end{abstract}

\section{Introduction}

Kikuchi-Fujimoto disease (KFD), sometimes referred to as histiocytic necrotizing lymphadenitis, is a self-limited disorder of unknown cause characterized by fever and tender lymphadenopathy, most commonly in young females between 20-30 years of age. Although it continues to be most common in the Asian population since its first description in Japan in 1972, it has a global distribution. Enlarged lymph nodes may be single or multiple - most commonly in the neck - but may also present in the axilla, and less commonly in deeper sites. ${ }^{1,2}$ Overall, involvement of retroperitoneal lymph nodes is rare, and only a few cases have been reported. ${ }^{3-5}$

Here, we report a patient of KFD presenting with fever of unknown origin (FUO), abdominal pain, and unusual retroperitoneal lymphadenopathy.

\section{Case Report}

A 50-year-old woman presented with a one-month history of cyclic fevers to $39.4^{\circ} \mathrm{C}$ and a diffuse erythematous rash. On initial presentation, she had generalized body aches, dysuria, and upper respiratory symptoms, and was believed to have a viral syndrome and urinary tract infection, for which she was treated with cephalexin. Although the dysuria resolved, she subsequently developed fevers, chills, and a new urticarial rash on both upper extremities and right thigh. She started a trial of hydroxyzine without improvement. Laboratory findings were significant for elevated $\mathrm{LDH}$ (448 U/L), ALT (41U/L), and AST (45U/L), however, hepatitis serologies were negative. Initial blood cultures detected growth of gram-positive rods in one out of four anaerobic bottles and was considered a contaminant. Repeat blood cultures were negative for growth. Interferon gamma release assay (Quantiferon Gold $\AA$ ) testing was negative for tuberculosis. Serologic testing was negative for rickettsia, parvovirus $\operatorname{IgM}$, cytomegalovirus IgM, coccidioidomycosis, Epstein-Barr virus IgM, and histoplasmosis. Many inflammatory markers were elevated, including C-reactive protein $(59.9 \mathrm{mg} / \mathrm{L})$, ferritin $(13,088 \mathrm{ng} / \mathrm{mL})$, and procalcitonin $(0.90 \mathrm{ng} / \mathrm{mL})$, and they continued to increase dramatically (e.g. ferritin peaking at $67,000 \mathrm{ng} / \mathrm{mL}$ ) until day 8 of her admission when there was a gradual downtrend. Testing for antineutrophil cytoplasmic antibody, antinuclear antibody, antidouble stranded DNA antibody, myeloperoxidase antibodies and proteinase B antibodies was negative. Computed tomography (CT) of the thorax and abdomen identified mildly enlarged spleen and liver, and multiple prominent upper abdominal, retroperitoneal, and pelvic lymph nodes, up to $1 \mathrm{~cm}$ in greatest dimension. A subsequent ${ }^{18} \mathrm{~F}$ flurodeoxyglucose positron emission tomography/CT (PET CT) scan detected increased activity in these lymph nodes, in particular, a conglomerate of multiple prominent peripancreatic lymph nodes exhibiting a standardized uptake value (SUV) of 24.4. The inguinal lymph nodes, while enlarged, had lower uptake values (maximum SUV of 2.6), and the spleen had a maximum SUV of 5.9. An abdominal ultrasound reported normal-appearing spleen, and liver with echogenicity typical of hepatic steatosis.

Bone marrow biopsy revealed no significant pathologic abnormalities, and biopsy of the skin rash showed a non-specific sparse perivascular and interstitial dermatitis. Although biopsy of the inguinal lymph
Correspondence: Russell K. Brynes, USC Department of Pathology, 2011 Zonal Ave., HMR 308 Los Angeles, CA 90033, USA.

Tel.: 323-409-7020 - Fax: 323-226-2686

E-mail: Brynes@med.usc.edu

Key words: Kikuchi-Fujimoto disease, histiocytic necrotizing lymphadenitis, retroperitoneal lymph node.

Contributions: JDH, MEV-L, IS and RKB examined the histolopathologic materials. $\mathrm{CF}$ and DIF managed the patient. JDH and RKB wrote the manuscript. All authors have contributed significantly to the conception, drafting, and analysis of this case report and review of literature, and approve of the final version for publication. All authors agree to be accountable for each aspect of this report.

Conflict of interest: The authors declare no conflict of interest.

Funding: None.

Ethics approval and consent to participate: This study was conducted ethically and in accordance with the institutional review board (IRB) of USC (University of Southern California) Health Sciences Campus and with the World Medical Association Declaration of Helsinki.

Availability of data and materials: Data and materials available within the text.

Received for publication: 2 November 2020 . Accepted for publication: 19 February 2021.

This work is licensed under a Creative Commons Attribution-NonCommercial 4.0 International License (CC BY-NC 4.0).

${ }^{\circ}$ Copyright: the Author(s), 2021

Licensee PAGEPress, Italy

Hematology Reports 2021; 13:9001

doi:10.4081/hr.2021.9001

node was entertained, due to the considerably lower SUV, it was determined likely to be low yield for diagnosis. Thus, despite their deep-seated nature, excision of peripancreatic lymph nodes with the highest SUV was performed. The biopsy specimen consisted of multiple enlarged lymph nodes, the largest measuring $5 \mathrm{~cm}$ in greatest dimension. Microscopic examination revealed partial effacement of the lymph node architecture by paracortical expansion with geographic foci of apoptotic necrosis, abundant karyorrhectic debris, numerous phagocytic histiocytes, rare plasmacytoid dendritic cells, and occasional histiocytes with crescent-shaped nuclei (Figure 1). Notably, plasma cells, neutrophils and eosinophils were absent. Surrounding areas 
of paracortical expansion were composed of a proliferation of small lymphocytes, scattered histiocytes, immunoblasts, and numerous small branching vessels. A rim of residual cortex contained predominantly primary follicles, and rare regressed germinal centers.

Immunohistochemical analysis was performed and supported the diagnosis of KFD. In the pathologic foci of paracortical expansion, the lymphocytes were largely CD3 positive, with a CD8 predominance. These cells also expressed TIA-1. CD20 positive lymphocytes were rare. The histiocytes, including those with crescentic nuclei, were positive for CD4, CD68, and CD163 (Figure 2A). Of note, the crescentic histiocytes were also positive for myeloperoxidase. Scattered CD123 positive plasmacytoid dendritic cells loosely surrounded the pathologic foci (Figure 2B). In-situ hybridization for detection of Epstein-Barr virus-encoded RNA was negative, and special stains for acid fast bacilli and fungal organisms were negative. Over the patient's 20-day hospital course, she was treated with ibuprofen and had gradual improvement of symptoms with supportive care. There have been no recurrent symptoms at six months of follow up.

\section{Discussion and Conclusions}

KFD is a rare, self-limited disease that is characterized by necrotizing histiocytic lymphadenopathy, often associated with localized pain and fever. It most commonly presents in the posterior cervical lymph nodes (60-90\% of cases), frequently with simultaneous involvement of axillary, and/or supraclavicular lymph nodes. ${ }^{6}$ Hepatosplenomegaly is uncommon. Extranodal involvement is rare, but when present, it is commonly cutaneous, presenting as rashes, nodules, or erythematous lesions. ${ }^{1,2,7,8}$

Since this entity was first described in 1972, many large case series and literature reviews $1,3,9,10$ have documented posterior cervical lymph nodes as the most common site of presentation. Extra-cervical nodal presentation is not uncommon, the abdomen being the most common extra-cervical site in one study with an incidence of abdominal cases as high as $20 \%$ of KFD cases. ${ }^{10}$ However, retroperitoneal involvement is rare, with only seven cases explicitly described as retroperitoneal since 1983 (Table 1). One study from Israel reported a uniquely different distribution of lymph node involvement, ${ }^{11}$ with cervical involvement in only $44 \%$ of 19 cases, generalized

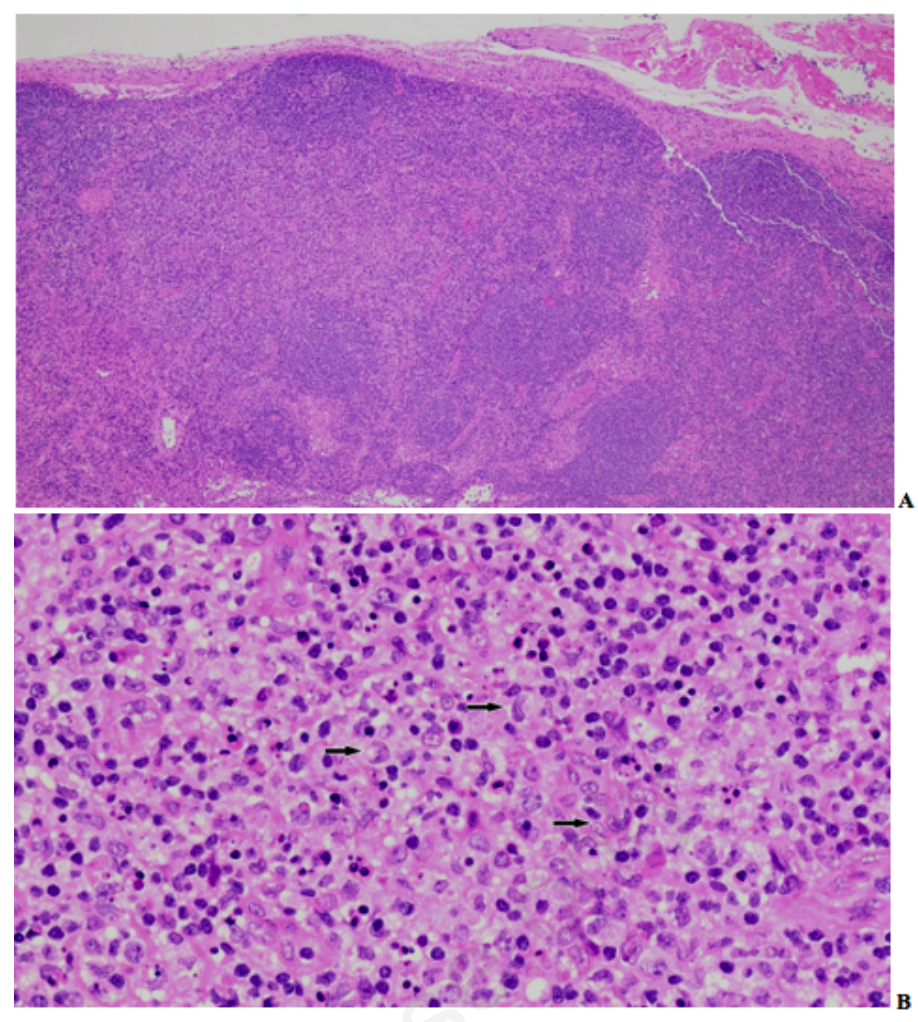

Figure 1. A) The largest lymph node contains patchy foci of extensive paracortical necrosis $(\mathrm{H} \& \mathrm{E}, 2 \mathrm{X})$. B) The necrotic area contains many histiocytes admixed with lymphocytes and extensive karyorrhectic nuclear debris. Many of these histiocytes display distinctive elongated, twisted, or crescentic nuclei (arrows). Of note, there are no neutrophils or plasma cells within the area of necrosis $(\mathrm{H} \& \mathrm{E}, 40 \mathrm{X})$.

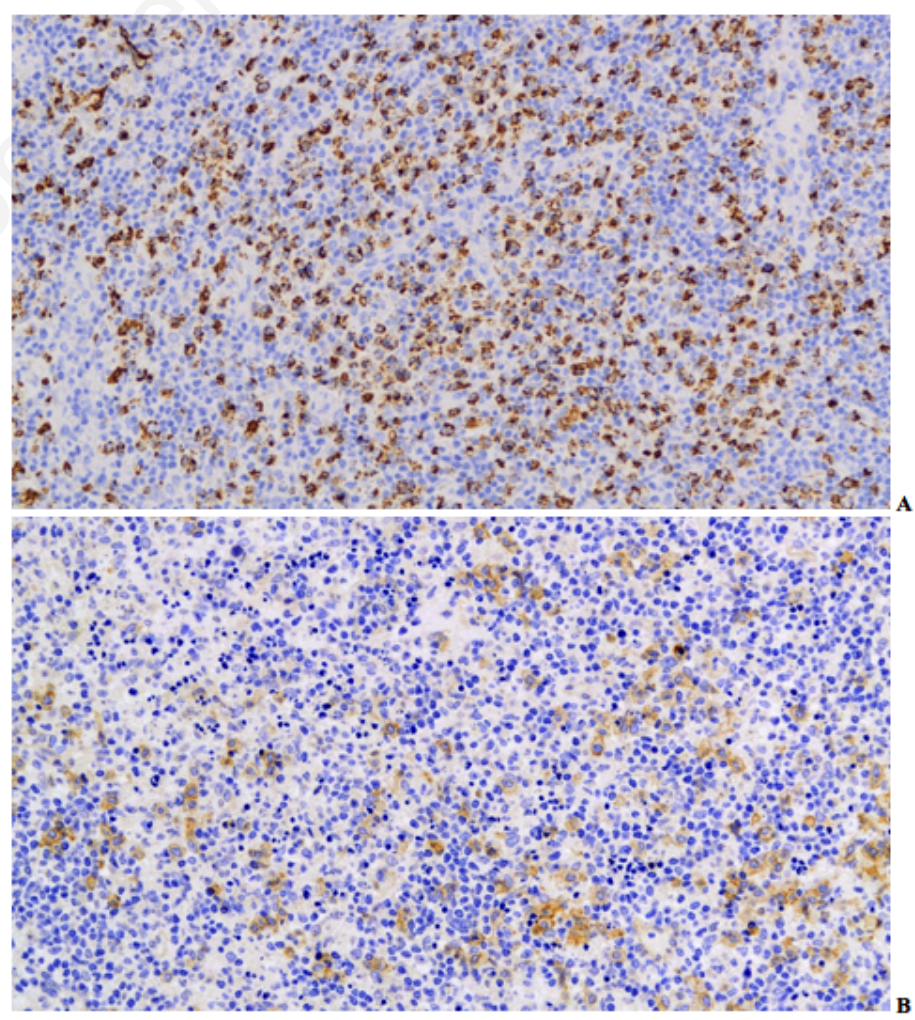

Figure 2. A) Histiocytes in the necrotic areas stain strongly for CD163 (Immunostain, 10X). B) CD123 staining identifies plasmacytoid dendritic cells infiltrating the margin of the necrotic focus (Immunostain, 10X). 
Table 1. Description of cases with retroperitoneal lymph node involvement.

\begin{tabular}{lcl} 
Reference & Total cases & Retroperitoneal cases \\
Turner et al. (1983) & 35 & 1 case (3\% of total): 24 -year old female, retroperitoneal along common iliac artery and pericaval area below right kidney \\
Kallam et al. (2016) & 1 & 1 case: 45 -year old female, retroperitoneal in subdiaphragmatic area \\
\hline Rimar et al. $(2010)$ & 19 & 4 cases (21\% of total): details not available \\
Vu et al. $(2016)$ & 1 & 1 case: 48 -year old male, retroperitoneal in right periceliac and peripancreatic area \\
\hline
\end{tabular}

lymphadenopathy in $26 \%$, and retroperitoneal involvement in $21 \%$; nevertheless, retroperitoneal cases worldwide are rarely reported. ${ }^{3-5}$ One particularly large English language literature review from 2007 analyzed 224 cases from 1991 to 2005, with the greatest number of cases coming from Taiwan (119 cases total), and found that cervical lymphadenopathy was overwhelmingly the most common presentation $(79 \%$ of cases). ${ }^{12}$ Simultaneous cervical and axillary involvement represented only $8 \%$ of cases, primary axillary involvement $5 \%$, and generalized lymphadenopathy $5 \%$. Other sites of involvement (listed as inguinal, mesenteric, and cutaneous lymph nodes) was exceedingly rare, and retroperitoneal involvement was not mentioned. Despite the increased availability of CT scans, these more recent studies still found cervical involvement to be the most common presentation, without an increase in cases of retroperitoneal involvement. ${ }^{10,11,13}$

Although painful lymphadenopathy and fever constitute the most common presentation, other symptoms include weight loss, nausea, night sweats, upper respiratory symptoms, headache, and weakness. These symptoms likely reflect the acute inflammatory state. While most patients have normal laboratory values, there may be mild anemia, slightly elevated erythrocyte sedimentation rate, and elevated C-reactive protein, serum lactate dehydrogenase, and aminotransferases. . $^{1,6}$

Imaging frequently demonstrates lymph nodes with increased size, some exceeding $4 \mathrm{~cm} .{ }^{13-15}$ Due to the acute or subacute presentation, aggressive lymphoma is typically considered in the differential diagnosis. While PET-CT scanning is a useful tool in the evaluation of lymphadenopathy, it is not specific for diagnosing malignancy, and indeed, increased uptake occurs in tuberculosis, sarcoidosis, and in reactive lymphadenopathies. In several studies with histologic correlation, PET-CT scans resulted in both false positive and false negative results of malignant diagnoses. ${ }^{16,17}$ Indeed, PET-CT scans of lymph nodes involved by KFD often demonstrate elevated SUVs, with uptake values as high as the upper 20 s, that overlap those seen in aggressive non-
Hodgkin lymphomas. ${ }^{13,15}$

With such a broad differential diagnosis, histologic examination is a necessity for accurate diagnosis. Classically, lymph nodes with KFD display paracortical necrotizing/karyorrhectic foci composed of increased histiocytes and immunoblasts admixed with small lymphocytes. Although a fine needle aspiration may be performed, due to its focal nature, diagnoses may be missed due to sampling error. Cases involving cervical lymph nodes may be diagnosed by core needle biopsy, ${ }^{18}$ however, due to the difficulty to access the retroperitoneal location in our case, excisional biopsy was performed. Indeed, involvement in our case was focal and likely would have been missed by core needle biopsy (Figure 1).

The etiology of KFD is unknown, and despite reports of patients with either concurrent viral infections or established autoimmune disease, a conclusive causative relationship has yet to be found. ${ }^{19,20}$ While it is typically a self-limited disease, the differential diagnosis is wide, including infectious causes, acute inflammation/autoimmune disorders, and non-Hodgkin lymphoma. The unusual retroperitoneal presentation in this patient contributed to the diagnostic challenge, as obtaining an adequate specimen for histopathological examination was difficult due to its deep-seated location. Although most infectious and autoimmune/acute inflammatory causes were excluded by laboratory testing, malignancy remained a major concern. With no medical intervention other than NSAID therapy, the patient showed improvement over her hospital course. Despite the unusual presenting site in this case, KFD should be considered in the differential diagnosis of patients presenting with fevers of unknown origin, lymphadenopathy, and associated serologic markers of acute inflammation.

\section{References}

1. Dumas G, Prendki V, Haroche J, et al. Kikuchi-Fujimoto disease: retrospective study of 91 cases and review of the literature. Medicine (Baltimore)
2014;93:372-82.

2. Pepe F, Disma S, Teodoro C, et al. Kikuchi-Fujimoto disease: a clinicopathologic update. Pathologica 2016;108:120-9.

3. Turner RR, Martin J, Dorfman RF. Necrotizing lymphadenitis. A study of 30 cases. Am J Surg Pathol 1983;7:11523.

4. Kallam A, Bierman PJ, Bociek RG. Kikuchi's disease masquerading as refractory lymphoma. J Oncol Pract 2016;12:94-6.

5. Vu D, Reddy S, Day L, et al. KikuchiFujimoto Disease: unusual presentation of rare disease. Clin Pract 2016;6:828.

6. Perry AM, Choi SM. Kikuchi-Fujimoto disease: a review. Arch Pathol Lab Med 2018;142:1341-6.

7. Atwater AR, Longley BJ, Aughenbaugh WD. Kikuchi's disease: case report and systematic review of cutaneous and histopathologic presentations. J Am Acad Dermatol 2008;59:130-6.

8. Kim JH, Kim YB, In SI, et al. The cutaneous lesions of Kikuchi's disease: a comprehensive analysis of 16 cases based on the clinicopathologic, immunohistochemical, and immunofluorescence studies with an emphasis on the differential diagnosis. Hum Pathol 2010;41:1245-54.

9. Pileri SA, Facchetti F, Ascani S, et al. Myeloperoxidase expression by histiocytes in Kikuchi's and Kikuchi-like lymphadenopathy. Am J Pathol 2001; 159:915-24.

10. Chen LC, Wang CJ, Chang YC, et al. Distribution of lymphadenopathy in patients with Kikuchi disease. J Microbiol Immunol Infect 2019:S1684.

11. Rimar D, Zisman D, Schendler Y, et al. Kikuchi Fujimoto disease in Israelmore than a pain in the neck. Semin Arthritis Rheum 2010;39:515-20.

12. Kucukardali Y, Solmazgul E, Kunter E, et al. Kikuchi-Fujimoto disease: analysis of 244 cases. Clin Rheumatol 2007;26:50-4.

13. Tsujikawa T, Tsuchida T, Imamura $Y$, et al. Kikuchi-Fujimoto disease: PET/CT assessment of a rare cause of cervical lymphadenopathy. Clin Nucl Med 
2011;36:661-4.

14. Kwon SY, Kim TK, Kim YS, et al. CT findings in Kikuchi disease: analysis of 96 cases. AJNR Am J Neuroradiol 2004;25:1099-102.

15. Zhang J, Dong MJ, Liu KF, et al. (18)FFluorodeoxyglucose positron emission tomography/computed tomography in patients with Kikuchi-Fujimoto disease: a nine-case series in China. Int $\mathrm{J}$ Clin Exp Med 2015;8:21034-43.

16. Tsukada T, Nakano T, Matoba M, et al. False-positive mediastinal lym- phadenopathy on 18F-fluorodeoxyglucose positron emission tomography and computed tomography after rectal cancer resection: a case report of thoracoscopic surgery in the prone position. Case Rep Oncol 2011;4:569-75.

17. Yu C, Xia X, Qin C, et al. Is SUV max helpful in the differential diagnosis of enlarged mediastinal lymph nodes? A pilot study. Contrast Media Mol Imaging 2018;2018:3417190.

18. Yu SC, Chen CN, Huang HI, et al. Diagnosis of Kikuchi-Fujimoto disease: a comparison between open biopsy and minimally invasive ultrasound-guided core biopsy. PLoS One 2014;9:e95886.

19. Chong Y, Kang CS. Causative agents of Kikuchi-Fujimoto disease (histiocytic necrotizing lymphadenitis): a metaanalysis. Int J Pediatr Otorhinolaryngol 2014;78:1890-97.

20. George TI, Jones CD, Zehnder JL, et al. Lack of human herpesvirus 8 and Epstein-Barr virus in Kikuchi's histiocytic necrotizing lymphadenitis. Human Pathol 2003;34:130-35. 